\title{
Are Cryptocurrencies a Safe Haven for Equity Markets? An International Perspective from the COVID-19 Pandemic.
}

\author{
Thomas Conlon ${ }^{\mathrm{a}}$, Shaen Corbet ${ }^{\mathrm{b}, \mathrm{c}}$, Richard J. McGee ${ }^{\mathrm{a}, *}$ \\ ${ }^{a}$ Smurfit Graduate School of Business, University College Dublin, Ireland. \\ ${ }^{b}$ DCU Business School, Dublin City University, Dublin 9, Ireland. \\ ${ }^{c}$ School of Accounting, Finance and Economics, University of Waikato, New Zealand.
}

\begin{abstract}
The COVID-19 pandemic provided the first widespread bear market conditions since the inception of cryptocurrencies. We test the widely mooted safe haven properties of Bitcoin, Ethereum and Tether from the perspective of international equity index investors. Bitcoin and Ethereum are not a safe haven for the majority of international equity markets examined, in fact increasing portfolio downside risk. Only investors in the Chinese CSI 300 index realized modest downside risk benefits, but only from small relative allocations to Bitcoin or Ethereum. As Tether successfully maintained its peg to the US dollar during the COVID-19 turmoil, it acted as a safe haven investment for all of the international indices examined. We caveat the latter findings with a warning that this dollar peg has not always been maintained, impairing the earlier downside risk hedging properties of Tether.
\end{abstract}

Keywords: Cryptocurrency; Bitcoin; Ethereum; Tether; Safe Haven; Downside Risk; COVID-19.

*Corresponding Author. E-mail: richard.mcgee@ucd.ie, Tel: +353-1-7164301

This publication has emanated from research conducted with the financial support of Science Foundation Ireland under Grant Number 16/SPP/3347 and 13/RC/2106 and 17/SP/5447. 


\section{Introduction}

The recent COVID-19 pandemic provided the first widespread bear market since the trading of cryptocurrencies began. In this article we examine the safe haven benefits of cryptocurrencies during the COVID-19 bear market, from the perspective of international equity index investors. Proponents of the view that cryptocurrency should be considered as an asset class, comparable to traditional financial assets such as oil and gold, have pointed towards hedging and diversification benefits as a key reason as to why these products will further expand and consolidate (Guesmi et al. (2019)). Some opponents have highlighted the relatively simplistic explanation of such relationships, derived within persistent evidence of broad product immaturity, substantial illegality and financial misappropriation (Corbet et al. (2019a,b)). Other research points out that sharp price increases and bubble-like behaviour has been driven by a small number of market agents (Gandal et al. (2018)). However, irrespective of the widespread issues identified, the recent COVID-19 pandemic has presented a unique opportunity to quantify the safe-haven properties central to the largest and most liquid cryptocurrencies, as identified through the reduction of downside risk of cryptocurrency-inclusive portfolios during this time. While considering such debate surrounding viability, future-proofing and both ethical and regulatory concern with this growing asset class, this research focuses specifically on diversification benefits to portfolio investors.

Such research builds on the concept of loss aversion (Tversky and Kahneman, 1991), which is of particular relevance in financial markets, where investors are often more concerned with avoiding losses than on any associated prospective gains (Hwang and Satchell, 2010). This loss aversion may motivate investors to seek out safe haven assets, which are uncorrelated or negatively correlated with traditional assets during periods of market turmoil (Baur and Lucey, 2010). Various safe haven assets have been established at short to medium horizons, including gold (Bredin et al., 2015), currencies (Ranaldo and Söderlind, 2010), long dated treasury bonds (Flavin et al., 2014) and, most recently, cryptocurrencies.

The growing popularity of cryptocurrencies has inspired numerous studies of their 
investment benefits, including their safe haven properties. Urquhart and Zhang (2019) look at the hedging and safe haven properties of Bitcoin at the hourly frequency and find that it acts as a hedge, diversifier and safe haven, respectively, for a range of international currencies. Shahzad et al. (2019) use a cross-quantilogram approach to define safe haven characteristics and find some evidence that Bitcoin, gold, and the commodity index are weak safe havens but that this behavior is timevarying. Guesmi et al. (2019) find that portfolio risk is found to be considerably reduced, through the inclusion of Bitcoin, relative to the risk of a portfolio comprising gold, oil and emerging market stocks alone.

Conversely, Smales (2019) finds that Bitcoin is more volatile, less liquid, and costlier to transact than other assets and suggests that these features should rule it out as safe haven asset. Klein et al. (2018) contrast the hedging and safe haven properties of gold and Bitcoin, finding that the latter correlates positively with downward moves in developed markets. The evidence for speculative behaviour in cryptocurrencies, manifesting in the form of bubbles (Corbet et al., 2018), may also shed doubt on their ability to act as a safe haven.

While results pre-COVID-19 are decidedly mixed, many of these studies found evidence supporting the hypothesis that cryptocurrencies had safe haven properties. Due to the absence of a bear market in the historical sample pre-COVID-19, this hypothesis was not tested under acute market conditions. Conlon and McGee (2020) investigate the safe haven properties of Bitcoin for a US investor investing domestically during the COVID-19 bear market and found that a portfolio allocation to Bitcoin increased rather than decreased downside risk exposure.

There are, however, reasons to believe that safe haven properties may vary internationally. One of the main candidate drivers put forward for the safe haven hypothesis in the literature is the independence of cryptocurrencies in monetary policy. Baker et al. (2016) develop an index of economic policy uncertainty (EPU, including monetary policy uncertainty) based on the measured frequency of related keywords in newspaper coverage. Local events such as elections have been shown to have a significant impact on both policy uncertainty and financial market uncertainty (see, e.g. Kelly et al., 2016; Goodell et al., 2020). There have been a number of 
papers investigating the relationship between cryptocurrency returns and EPU (see, e.g. Demir et al., 2018; Wang et al., 2019a; Wu et al., 2019; Fang et al., 2019). Wang et al. (2020), for example, find that Bitcoin returns around the highest economic policy uncertainty days (as measured by local versions of the EPU index of Baker et al., 2016) are significantly greater than those around the lowest EPU days. Aysan et al. (2019) investigate the impact of global geopolitical risk (GPR) on Bitcoin. They find that GPR has predictive power on returns and price volatility of Bitcoin. Negative changes in GPR significantly lead to greater Bitcoin returns. Bouri et al. (2017a) examine whether Bitcoin can hedge global uncertainty, measured by the first principal component of the VIXs of 14 developed and developing equity markets. They find that Bitcoin does act as a hedge against uncertainty: it reacts positively to uncertainty at both higher quantiles and shorter frequency movements of Bitcoin returns. As policy uncertainty (and monetary policy uncertainty) are country-specific, these studies motivate an analysis of the safe haven properties of cryptocurrencies internationally.

In prior work, specifically in the context of international safe havens, Mensi et al. (2020) examine the co-movements between Bitcoin and the Dow Jones World Stock Market Index; regional Islamic stock markets; and Sukuk markets, using wavelet transformation techniques. They find evidence of benefits from diversification with Bitcoin, but these are found to be smaller for longer term investors (order of months) compared to short-term investors (order of days). Kliber et al. (2019) use a multivariate stochastic volatility model with dynamic conditional correlation to examine the time-varying hedging and safe haven properties of Bitcoin. Bitcoin is found to be a weak hedge in all markets when investment in US dollars is considered and a safe haven in Venezuela (with investment in bolivars). Bouri et al. (2017b) use dynamic conditional correlations and find that Bitcoin has hedge and safe haven properties against Asia Pacific stocks. Assessing a wide range of cryptocurrencies, Wang et al. (2019b) find that digital currencies act as a safe haven for most international indices examined.

In this paper, we consider the international safe haven properties of cryptocurrencies through a popular methodology in the safe haven literature, specifically by ex- 
amining whether there is a reduction in downside risk through pairing international equity index investments with portfolio allocations to individual cryptocurrencies (candidate cryptocurrencies include: Bitcoin, Ethereum ${ }^{1}$ and Tether). Downside risk is estimated using the approach of modified value at risk and modified conditional value at risk proposed by Favre and Galeano (2002), capturing higher order moments frequently attributed to financial returns. We consider a range of international equity indices including: MSCI World; SESP 500 (US); FTSE 100 (UK); FTSE MIB (Italy); IBEX (Spain) and CSI 300 (China). These indices are selected as they represent share prices in countries most seriously impacted by the pandemic at the onset of the COVID-19 bear market, in late February 2020.

We find that Bitcoin and Ethereum are not safe havens for almost all of the indices during the COVID-19-19 market turmoil. Our findings are in contrast to Feng et al. (2018), who find that the tail risk of Bitcoin is independent of returns in stock indices over a period ending in August 2017. In keeping with the literature, in rolling window analysis, we find that cryptocurrencies may act as a hedge during certain market periods. Consistent with Bouri et al. (2017b), who find that Bitcoin has hedge and safe haven properties for Asia Pacific stocks pre-COVID-19, we find some limited evidence of safe haven properties relative to the CSI 300 index across the sample range tested and during the COVID-19 crisis. These findings only hold, however, for allocations of up to $16 \%$ to Bitcoin and $14 \%$ to Ethereum. For larger allocations we again observe increasing relative portfolio downside risk for CSI 300 investors. We also find that the stable coin Tether has safe haven properties across all indices examined during the COVID-19 bear market, but may be redundant as an asset, given its peg to the US dollar. ${ }^{2}$ We provide evidence that this peg is not consistently maintained over the period examined, impairing the consistency of any downside risk hedging properties.

The paper is organized as follows: Section 2 outlines the methodological approach

\footnotetext{
${ }^{1}$ Beneki et al. (2019) find that Bitcoin and Ethereum are linked through a volatility transmission channel.

${ }^{2}$ Tether has additional costs relative to holding cash such as a $0.1 \%$ deposit fee and a maximum of $0.1 \%$ or $\$ 1,000$ withdrawal fee into fiat currency.
} 
employed to quantify downside risk. In Section 3, we detail the data under consideration. Empirical findings are described in Section 4, while Section 5 concludes.

\section{Methodology}

\subsection{Downside Risk Measurement}

Olsen (1997) highlights that a primary concern of investors is the potential for large losses stemming from an investment. Such downside risk has been shown to be priced in the market place, with investors requiring higher returns on stocks exhibiting greater downside risk (Ang et al., 2006). In this paper, we focus on value at risk (VaR) and the related conditional value at risk (CVAR), also referred to as expected shortfall, to measure potential losses in a portfolio over a given time horizon (Conlon and Cotter, 2013). While other measures of crash risk have also been proposed in the context of cryptocurrencies (Kalyvas et al., 2019), the methods detailed here are appropriate for frequently examined horizons of importance to investors.

Two-moment VaR is appropriate to estimate downside risk for a portfolio with returns strictly distributed according to a normal distribution. Setting a particular confidence interval, VaR is defined as the maximum expected loss on a portfolio over a given time horizon. Under the assumption of normally distributed returns, two moment VaR is estimated using

$$
\operatorname{VaR}_{p}(1-\alpha)=\mu_{p}-\sigma_{p} z(\alpha)
$$

$\alpha$ is the confidence interval considered, while $z(\alpha)$ is the $\alpha$ quantile of the standardized distribution. $\mu_{p}$ and $\sigma_{p}$ are the mean and standard deviation of portfolio returns respectively. For two-moment VaR, the downside risk of an asset is just a constant multiple of the standard deviation of asset returns.

While frequently applied in practise, two moment VaR may not adequately capture the potential for large losses when the distribution of returns is not normal. Cryptocurrencies have been shown previously to have returns presenting both skewness and excess kurtosis (Conlon and McGee, 2020; Eross et al., 2019), implying 
that two moment VaR is insufficient as a measure of their downside risk. Using the Cornish-Fisher expansion to adjust the quantiles of the distribution to account for higher-order moments related to skewness and excess kurtosis, we employ the four moment VaR first proposed by Favre and Galeano (2002). Four moment VaR is based upon an approximation for the quantile of the distribution, cut off at the fourth moment, and given by:

$$
\begin{aligned}
& \hat{Z}\left(\alpha, S_{p}, K_{p}\right)=z(\alpha)+\frac{1}{6}\left(z(\alpha)^{2}-1\right) S_{p}+\ldots \\
& \frac{1}{24}\left(z(\alpha)^{3}-3 z(\alpha)\right) K_{p}-\frac{1}{36}\left(2 z(\alpha)^{3}-5 z(\alpha)\right) S_{p}^{2}
\end{aligned}
$$

$S_{p}$ and $K_{p}$ are the skewness and kurtosis of portfolio $P$, while $z(\alpha)$ is the $\alpha$ quantile of the standard normal distribution. Four moment modified VaR is then given by:

$$
\operatorname{MVaR} R_{p}(1-\alpha)=\mu_{p}-\sigma_{p} \hat{Z}\left(\alpha, S_{p}, K_{p}\right)
$$

This adjusts the two-moment VaR (Equation 1) to account for distributional characteristics commonly found in financial time series.

CVAR looks to capture the loss expectation, conditional on the loss exceeding the modified VaR, $M V a R_{p}$. Modified CVaR is calculated as a function of modified VaR:

$$
M C V a R_{p}=\mathrm{E}\left(R_{p} \mid R_{p}>M V a R_{p}\right)
$$

where $R_{p}$ is the negative log return. As there is no simple analytical way to estimate modified value-at-risk, a numerical approach is employed, taking an average of $M V a R_{p}$ across the range of quantiles greater than $1-\alpha$.

To understand the economic impact of allocating a proportion of wealth to cryptocurrencies, we use the approach of relative portfolio downside risk (Conlon and McGee, 2020; Bredin et al., 2017). This is estimated by taking the portfolio downside risk with an allocation to a cryptocurrency relative to a portfolio holding only the equity index under consideration. For MVaR (MCVaR), this is given by $\frac{M V a R_{\text {mix }}}{M V a R_{\text {equity }}}$ $\left(\frac{M C V a R_{\text {mix }}}{M C V a R_{\text {equity }}}\right)$, where $M V a R_{m i x}$ and $M C V a R_{m i x}$ are downside risk measures repre- 
senting the mix portfolio containing an equity index and a cryptocurrency, respectively.

\section{Data}

The focus of this paper is isolate the portfolio risk implications of an allocation to cryptocurrencies for a US investor holding a position in a range of international equity markets. Three cryptocurrencies are studied, namely Bitcoin, Ethereum and Tether. Bitcoin, the best known, mostly widely traded cryptocurrency also has the largest market capitalization. Ethereum is a decentralized computing platform, from which the cryptocurrency Ether, commonly known as Ethereum, is issued as a reward for mining nodes. Finally, Tether is a stable coin, pegged to the US Dollar, meaning that it should maintain a 1-to-1 ratio with the U.S. dollar. This link is, however, not guaranteed by the issuer.

Daily data on the cryptocurrencies, Bitcoin, Ethereum and Tether is gathered from Coinmetrics, using their CM reference rates. This paper meets the critique for cryptocurrency data of Alexander and Dakos (2020), as it is formed using a methodology which adheres to the International Organisation of Securities Commissions (IOSCO) Principles for Financial Benchmarks. The objective of the IOSCO Principles is to "create an overarching framework of principles for benchmarks used in financial markets". All cryptocurrencies are examined in US Dollar terms.

We consider the portfolio downside risk implications for a variety of international markets, with a focus on countries known to have been impacted severely by the COVID-19 pandemic. The MSCI World Index is taken as representative of world equity markets, while the S\&P 500 is selected to illustrate findings for US Markets. The FTSE 100, FTSE MIB, IBEX and CSI 300 indices are selected to represent the equity investment opportunity set for the United Kingdom, Italy, Spain and China, respectively. All indices are priced in US Dollars and daily logarithmic returns are calculated.

The inception date for each of the Cryptocurrencies differs, resulting in different data availability. Price data for Bitcoin are available from April 2010 through April 2020, data relating to Ethereum are available from August 2015 through April 2020, 
while data on Tether are from October 2014 through April 2020. In order to isolate the impact of the COVID-19 pandemic, a common period from 11 ${ }^{\text {th }}$ April 2019 to $9^{\text {th }}$ April 2020 is examined. This period captures the initial bear market in equities associated with the COVID-19 pandemic. ${ }^{3}$ Finally, we assess the dynamic downside risk hedging ability of cryptocurrencies using a 1 year moving-window.

\section{Empirical Findings}

Summary statistics pertaining to our sample are presented in Table 1. We examine characteristics over the period April 2010 - April 2020 for Bitcoin and each of the indices. Results for Ethereum and Tether are presented from the first point at which traded prices exist. Bitcoin and Ethereum are found to have considerably higher returns and standard deviation than any of the indices examined. While Bitcoin is negatively skewed, Ethereum has positive skewness, in contrast to the equity indices considered. The maximum one-day loss for Bitcoin and Ethereum are $-66.5 \%$ and $-56.56 \%$ respectively. The range of one-day losses for equity indices is from $-9.42 \%$ (CSI 300) to $-19.29 \%$ (FTSE MIB). The differential characteristics of Tether are notable, presenting an average return of almost zero and a standard deviation of $26 \%$, larger than the majority of equity indices examined. Given the purported peg with the US dollar, it is remarkable that Tether exhibits a maximum one day loss of $-14.75 \%$.

We focus upon the most recent one year period, which incorporates the ongoing COVID-19 related market turmoil. Over this period, only Bitcoin and Ethereum display returns for which the mean return is positive, but with a relatively large standard deviation. Each of the equity indices display negative returns ranging from $-3.48 \%$ for the S\&P 500 to $-31.62 \%$ for the IBEX. During this one year interval, both Bitcoin and Ethereum have a maximum one day loss of $-47 \%$ and $-56.6 \%$ respectively. For Tether, the position is more benign, with a maximum one day loss of $-2.42 \%$.

\footnotetext{
${ }^{3} \mathrm{~A}$ bear market is often defined as one where the stock market drops by more than $20 \%$ from a recent high.
} 


\subsection{Unconditional Downside Risk Estimates}

In Table 2, we provide an initial assessment of the downside risk exposure of each index alone and for a portfolio with a $10 \%$ allocation to the cryptocurrency and the remaining $90 \%$ to the index. Downside risk is estimated using both MVaR and MCVaR at a $1 \%$ and $5 \%$ confidence level.

For both confidence levels, the Italian MIB index presents the highest MVaR and MCVaR among the equity indices examined. Over the most recent one year period, the MVaR for the MIB is $15.48 \%$ at a $1 \%$ confidence level. Creating a portfolio consisting of 10\% Bitcoin and $90 \%$ equity increases downside risk for all indices with the exception of the CSI 300. For example, the 1\% MVaR for the MIB increases from $7.90 \%$ to $9.23 \%$ during $2010-2020$, an increase of $16.8 \%$. In contrast, combining Bitcoin with the CSI 300 results in a decrease in MVaR from $4.77 \%$ to $4.32 \%$.

We can directly compare downside risk for portfolios containing Ethereum and Tether with index-only investments during the 2019-2020 period. For Ethereum, the only evidence for a reduction in downside risk is again observed for the CSI 300. The most substantial increase is found for the FTSE 100, which has an MVaR of $11.47 \%$ at a $1 \%$ confidence level, but increases by $28.9 \%$ when combined in a portfolio consisting of $10 \%$ Ethereum with the remaining $90 \%$ allocated to the FTSE 100. Tether provides some safe haven characteristics over this period. For each of the equity indices, we find that an allocation to Tether results in a reduction in downside risk. This alone is insufficient to confirm the safe haven properties of Tether. If a firm peg between Tether and the US dollar exists, a 10\% allocation is equivalent to $10 \%$

of the portfolio being unallocated and should result in a $10 \%$ decrease in downside risk. We next test this and the proportional reduction in downside risk across all assets for a range of different allocation weights.

\subsection{Cryptocurrency Allocation Weights}

Figure 1 details the reduction in MVaR and MCVaR for a set of Bitcoin allocation weights, relative to holding only the equity index over the period 2010-2020. Results are emphatic. Any allocation to Bitcoin results in increased MVaR and MCVaR for a portfolio consisting of Bitcoin combined with the MSCI World, S\&P 500, FTSE 
100, FTSE MIB and IBEX, relative to holding only the respective index. The CSI 300 is the only index for which diversifying with Bitcoin results in a reduction in downside risk, and there only for an allocation of up to $16 \%$. These findings indicate that Bitcoin does not act as a consistent safe haven for international equity indices, with allocations generally resulting in increased downside risk.

Similar conclusions are found for Ethereum over the period August 2015 through April 2020, as detailed in Figure 2. As the allocation to Ethereum is increased, portfolio MVaR and MCVaR increases relative to holding just the equity index for the MSCI World, S\&P 500, FTSE 100, FTSE MIB and IBEX. Ethereum provides some downside risk diversification for the CSI 300 for allocations of up to $14 \%$. As the allocation increases beyond this point, relative portfolio risk for the CSI 300 accelerates considerably. For a 50\% allocation to Ethereum, relative portfolio MVaR is 2.59 for the CSI 300 portfolio compared with 1.50 for the S\&P 500. Our findings highlight that Ethereum also does not have strong safe haven properties for international equity indices. For most allocation weightings Ethereum results in increased downside risk when combined with traditional equity markets.

Finally, we assess whether an allocation to Tether helps in reducing portfolio downside risk between October 2014 and April 2020. Tether was designed such that each coin is worth one US dollar. Worries about credit risk or the volume of currency underpinning the cryptocurrency may, however, result in Tether trading at less than one US dollar. This, in turn, may impact any potential as a safe haven. This is borne out in our findings, Figure 3. If Tether were to act as a US dollar equivalent investment, portfolio downside risk should decrease in a linear fashion for larger allocations. In fact, relative downside risk decreases for allocations of between $30 \%$ and $50 \%$, depending upon the equity index considered. For larger allocations, relative downside risk increases. With the exception of the CSI 300, downside risk declines one-for-one for each Tether allocation unit for weights of up to $35 \%$. The increasing relative downside risk beyond this point is attributed to the large one-day maximum loss associated with Tether of $14.75 \%$, which occurred on $5^{\text {th }}$ May 2015. Large daily losses such as these have not been unusual in the case of Tether, with losses of greater than $10 \%$ occurring on 5 occasions since 2014. Beyond its consideration as a safe 
haven, these losses highlight additional investment risks, most likely associated with credit risk concerns, for investors in Tether.

\subsection{Moving Window Analysis}

In this section, we examine the relative downside risk of a portfolio consisting of $10 \%$ cryptocurrency combined with a $90 \%$ allocation to the relevant equity index using a moving window of 1 year. This analysis allows us to assess whether cryptocurrencies were a hedge for equity markets during the relatively benign period up to the beginning of the COVID-19 market crisis. This may help in reconciling the previous evidence that cryptocurrencies, especially Bitcoin, have sometimes acted as a hedge for equity markets (Shahzad et al., 2019; Chan et al., 2019; Bouri et al., 2017b).

The downside risk reduction potential for Bitcoin over time is examined in Figure 4. Throughout the plots, a relative risk reduction below one indicates that a portfolio containing 10\% cryptocurrency and $90 \%$ equity has lower downside risk than a portfolio containing a full $100 \%$ allocation to the relevant equity index. Taking the FTSE 100 as an example, only on limited occasions is portfolio downside risk, whether measured by MVaR or MCVaR, reduced by allocating to Bitcoin. Over the sample considered, MVaR is increased by an average of $24.77 \%$ by allocating $10 \%$ to Bitcoin, while MCVaR is increased by $44.4 \%$. Only for a brief period from June 2016 through October 2017 does Bitcoin consistently reduce portfolio downside risk for the FTSE 100. With the exception of the CSI 300, similar findings are evident for the other indices examined. The CSI 300 shows some evidence of downside risk reduction since 2015, coinciding with the period from which the Bitcoin market has been suggested as being most efficient (Conlon and McGee, 2019; Urquhart, 2016).

Similar findings are evident for Ethereum, Figure 5. Some early evidence for risk reduction is found for most markets, coinciding with a similar brief period where Bitcoin acted as a hedge for equity markets. From 2018 onward, only limited evidence that Ethereum helps in reducing portfolio downside risk is found. In keeping with earlier findings, a portfolio consisting of a $10 \%$ allocation to Ethereum and $90 \%$ to the CSI 300 has lower downside risk post 2018 than when the latter is held alone. 
This finding is also evident in the period incorporating the COVID-19 crisis, but a noteworthy increase in portfolio risk is evident across all equity indices during this phase. This indicates that Ethereum has limited safe haven properties and a small allocation may increase portfolio downside risk in many circumstances.

In Figure 6, we examine the downside risk implications for a portfolio diversified with a $10 \%$ allocation to Tether. If Tether has a consistent peg to the US dollar, a $10 \%$ allocation should result in a stable $10 \%$ reduction in downside risk at all points in time. The level of downside risk reduction is not found to be static and fluctuates around 0.90. In the case of the MSCI World index, allocating to Tether results in increased portfolio risk in the period from September 2017 to February 2018. This highlights some underlying risks for investors using Tether as a safe haven, linked to the previously described large one-day downward price moves in the cryptocurrency. Tether retained its peg to the US dollar during the COVID-19 crisis, however, with only very limited increases in downside risk observed relative to the expected reduction to 0.90 . For example, a portfolio with a $10 \%$ allocation to Tether had a reduction of 0.907 (0.906) in MCVaR (MVaR) relative to one holding only the S\&P 500.

\section{Conclusion}

While cryptocurrencies are frequently part of the discussion regarding safe haven assets, previous empirical research on their relevance has lacked a period of significant turmoil in traditional markets such as equities. This paper considers the downside risk reduction properties of three cryptocurrencies, Bitcoin, Ether and Tether, during the initial bear market period associated with the COVID-19 crisis. We examine downside risk reduction for six international equity markets, shedding new light on the safe haven properties of cryptocurrencies for international investors.

Bitcoin and Ethereum are not, in general, found to act as a safe haven for international equity markets. We provide evidence of increased downside risk for portfolios consisting of any allocation to these two assets relative to holding the underlying equity index in isolation. An exception is for the CSI 300 index, where allocations of up to $16 \%$ to Bitcoin and $14 \%$ to Ethereum may reduce downside risk. Above these 
thresholds, however, we observe a marked relative increase in downside risk. Tether is found to act as a safe haven over the most recent period including the COVID-19 crisis. Such downside risk hedging properties are not, however, found to be consistent over time, due to large short-term historical losses in Tether, a consequence of an unstable peg with the US dollar. This raises a number of substantial questions as to why an investor would use Tether to hedge as opposed to the US dollar, particularly due to the added counter-party, technological, security and liquidity risk, along with further stability issues sourced in the variation of the stability of the peg during periods of exceptional financial crisis.

While evidence of cryptocurrency and safe-haven benefits have been previously identified, this research confirms that such price-based traits failed to manifest during periods of extreme financial market volatility resulting from the outbreak of the COVID-19 pandemic. Therefore, while there exist in excess of five thousand international cryptocurrencies, the three largest and most liquid did not provide a diversification channel during extreme financial crisis. While considering the collapse of international oil prices during the first quarter of 2020, and the existence of negative yields in high-grade, sovereign bond markets, portfolio investors have struggled substantially to identify safe havens during the period surrounding the pandemic. While gold has provided some portfolio safety, this research finds that cryptocurrencies did not offer any substantial benefit. 


\section{References}

Alexander, C., Dakos, M. (2020). A critical investigation of cryptocurrency data and analysis. Quantitative Finance, 20(2), 173-188.

Ang, A., Chen, J., Xing, Y. (2006). Downside risk. The Review of Financial Studies, 19(4), 1191-1239.

Aysan, A.F., Demir, E., Gozgor, G., Lau, C.K.M. (2019). Effects of the geopolitical risks on Bitcoin returns and volatility. Research in International Business and Finance, 47, 511-518.

Baker, S.R., Bloom, N., Davis, S.J. (2016). Measuring economic policy uncertainty. The Quarterly Journal of Economics, 131(4), 1593-1636.

Baur, D.G., Lucey, B.M. (2010). Is gold a hedge or a safe haven? An analysis of stocks, bonds and gold. Financial Review, 45(2), 217-229.

Beneki, C., Koulis, A., Kyriazis, N.A., Papadamou, S. (2019). Investigating volatility transmission and hedging properties between Bitcoin and Ethereum. Research in International Business and Finance, 48, 219-227.

Bouri, E., Gupta, R., Tiwari, A.K., Roubaud, D. (2017a). Does Bitcoin hedge global uncertainty? Evidence from wavelet-based quantile-in-quantile regressions. Finance Research Letters, 23, 8795.

Bouri, E., Molnár, P., Azzi, G., Roubaud, D., Hagfors, L.I. (2017b). On the hedge and safe haven properties of Bitcoin: Is it really more than a diversifier? Finance Research Letters, 20, 192-198.

Bredin, D., Conlon, T., Potì, V. (2015). Does gold glitter in the long-run? Gold as a hedge and safe haven across time and investment horizon. International Review of Financial Analysis, 41, $320-328$.

Bredin, D., Conlon, T., Potì, V. (2017). The price of shelter - Downside risk reduction with precious metals. International Review of Financial Analysis, 49, 48-58.

Chan, W.H., Le, M., Wu, Y.W. (2019). Holding Bitcoin longer: The dynamic hedging abilities of Bitcoin. The Quarterly Review of Economics and Finance, 71, 107-113.

Conlon, T., Cotter, J. (2013). Downside risk and the energy hedger's horizon. Energy Economics, 36, 371-379. 
Conlon, T., McGee, R.J. (2019). Betting on Bitcoin: Does gambling volume on the blockchain explain Bitcoin price changes? Economics Letters, 108727.

Conlon, T., McGee, R.J. (2020). Safe Haven or Risky Hazard? Bitcoin during the Covid-19 Bear Market. Bitcoin during the Covid-19 Bear Market (March 24, 2020).

Corbet, S., Lucey, B., Yarovaya, L. (2018). Datestamping the Bitcoin and Ethereum bubbles. Finance Research Letters, 26, 81-88.

Corbet, S., Lucey, B., Urquhart, A., Yarovaya, L. (2019a). Cryptocurrencies as a financial asset: A systematic analysis. International Review of Financial Analysis, 62, 182-199.

Corbet, S., Cumming, D.J., Lucey, B.M., Peat, M., Vigne, S.A. (2019b). The destabilising effects of cryptocurrency cybercriminality. Economics Letters, 108741.

Demir, E., Gozgor, G., Lau, C.K. M., Vigne, S.A. (2018). Does economic policy uncertainty predict the Bitcoin returns? An empirical investigation. Finance Research Letters, 26, 145-149.

Eross, A., McGroarty, F., Urquhart, A., Wolfe, S. (2019). The intraday dynamics of Bitcoin. Research in International Business and Finance, 49, 71-81.

Fang, L., Bouri, E., Gupta, R., Roubaud, D. (2019). Does global economic uncertainty matter for the volatility and hedging effectiveness of Bitcoin? International Review of Financial Analysis, 61, 29-36.

Favre, L., Galeano, J.-A. (2002). Mean-modified value-at-risk optimization with hedge funds. The Journal of Alternative Investments, 5(2), 21-25.

Feng, W., Wang, Y., Zhang, Z. (2018). Can cryptocurrencies be a safe haven: A tail risk perspective analysis. Applied Economics, 50(44), 4745-4762.

Flavin, T.J., Morley, C.E., Panopoulou, E. (2014). Identifying safe haven assets for equity investors through an analysis of the stability of shock transmission. Journal of International Financial Markets, Institutions and Money, 33, 137-154.

Gandal, N., Hamrick, J.T., Moore, T., Oberman, T. (2018). Price manipulation in the Bitcoin ecosystem. Journal of Monetary Economics, 95, 86-96.

Goodell, J.W., McGee, R.J., McGroarty, F. (2020). Election uncertainty, economic policy uncertainty and financial market uncertainty: A prediction market analysis. Journal of Banking $\mathcal{E}$ Finance, 110, 105684. 
Guesmi, K., Saadi, S., Abid, I., Ftiti, Z. (2019). Portfolio diversification with virtual currency: Evidence from bitcoin. International Review of Financial Analysis, 63, 431-437.

Hwang, S., Satchell, S.E. (2010). How loss averse are investors in financial markets? Journal of Banking $\mathscr{G}$ Finance, 34(10), 2425-2438.

Kalyvas, A., Papakyriakou, P., Sakkas, A., Urquhart, A. (2019). What drives Bitcoin's price crash risk? Economics Letters, 108777.

Kelly, B., Pástor, L., Veronesi, P. (2016). The price of political uncertainty: Theory and evidence from the option market. The Journal of Finance.

Klein, T., Thu, H.P., Walther, T. (2018). Bitcoin is not the New Gold-A comparison of volatility, correlation, and portfolio performance. International Review of Financial Analysis, 59, 105-116.

Kliber, A., Marszałek, P., Musiałkowska, I., Świerczyńska, K. (2019). Bitcoin: safe haven, hedge or diversifier? Perception of bitcoin in the context of a country's economic situation - a stochastic volatility approach. Physica A: Statistical Mechanics and its Applications, 524, 246-257.

Mensi, W., Rehman, M.U., Maitra, D., Al-Yahyaee, K. H., Sensoy, A. (2020). Does Bitcoin comove and share risk with Sukuk and world and regional Islamic stock markets? Evidence using a time-frequency approach. Research in International Business and Finance, 101230.

Olsen, R.A. (1997). Investment risk: The experts' perspective. Financial Analysts Journal, 53(2), $62-66$.

Ranaldo, A., Söderlind, P. (2010). Safe haven currencies. Review of Finance, 14(3), 385-407.

Shahzad, S.J.H., Bouri, E., Roubaud, D., Kristoufek, L., Lucey, B. (2019). Is Bitcoin a better safe-haven investment than gold and commodities? International Review of Financial Analysis, 63, 322-330.

Smales, L.A. (2019). Bitcoin as a safe haven: Is it even worth considering? Finance Research Letters, 30, 385-393.

Tversky, A., Kahneman, D. (1991). Loss aversion in riskless choice: A reference-dependent model. The Quarterly Journal of Economics, 106(4), 1039-1061.

Urquhart, A. (2016). The inefficiency of Bitcoin. Economics Letters, 148, 80-82.

Urquhart, A., Zhang, H. (2019). Is Bitcoin a hedge or safe haven for currencies? An intraday analysis. International Review of Financial Analysis, 63, 49-57. 
Wang, G.-J., Xie, C., Wen, D., Zhao, L. (2019a). When Bitcoin meets economic policy uncertainty (EPU): Measuring risk spillover effect from EPU to Bitcoin. Finance Research Letters, 31.

Wang, P., Zhang, W., Li, X., Shen, D. (2019b). Is cryptocurrency a hedge or a safe haven for international indices? A comprehensive and dynamic perspective. Finance Research Letters, 31, $1-18$.

Wang, P., Li, X., Shen, D., Zhang, W. (2020). How Does Economic Policy Uncertainty Affect the Bitcoin Market? Research in International Business and Finance, 101234.

Wu, S., Tong, M., Yang, Z., Derbali, A. (2019). Does gold or Bitcoin hedge economic policy uncertainty? Finance Research Letters, 31, 171-178. 


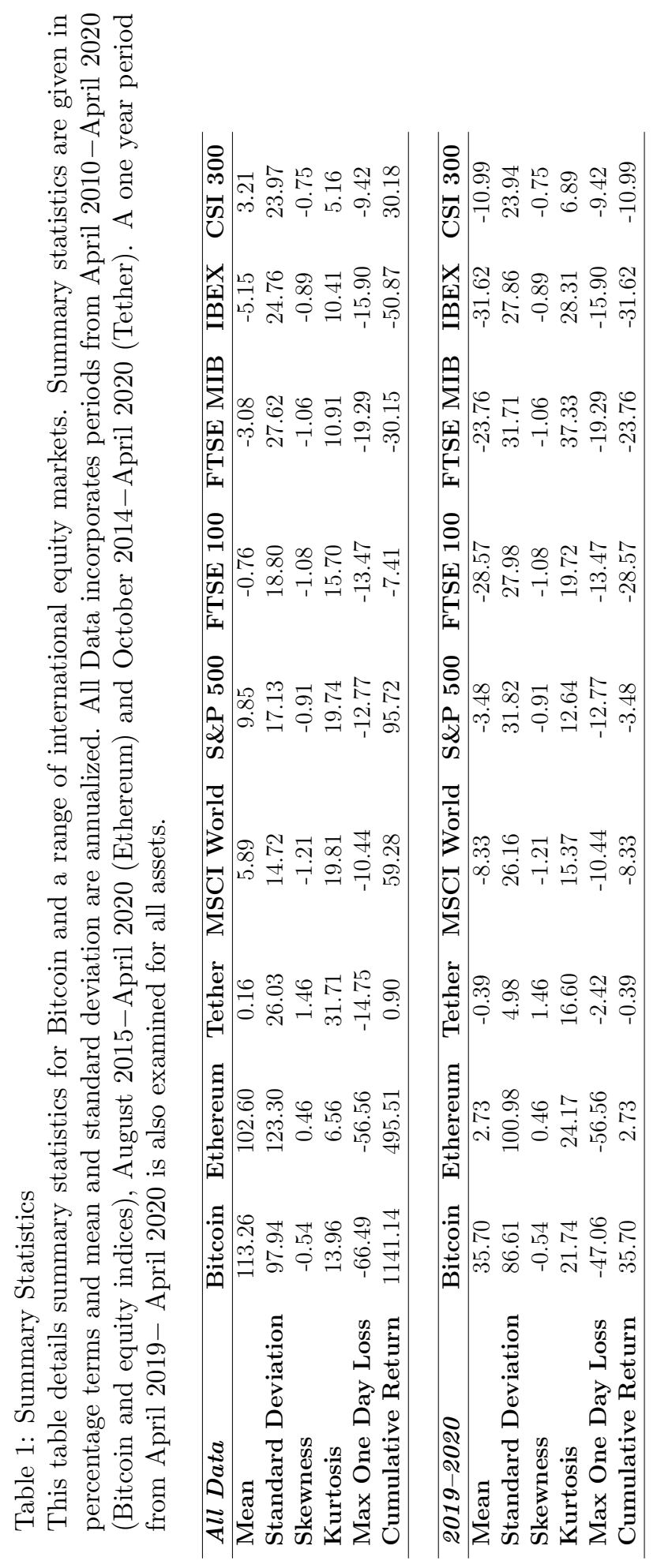




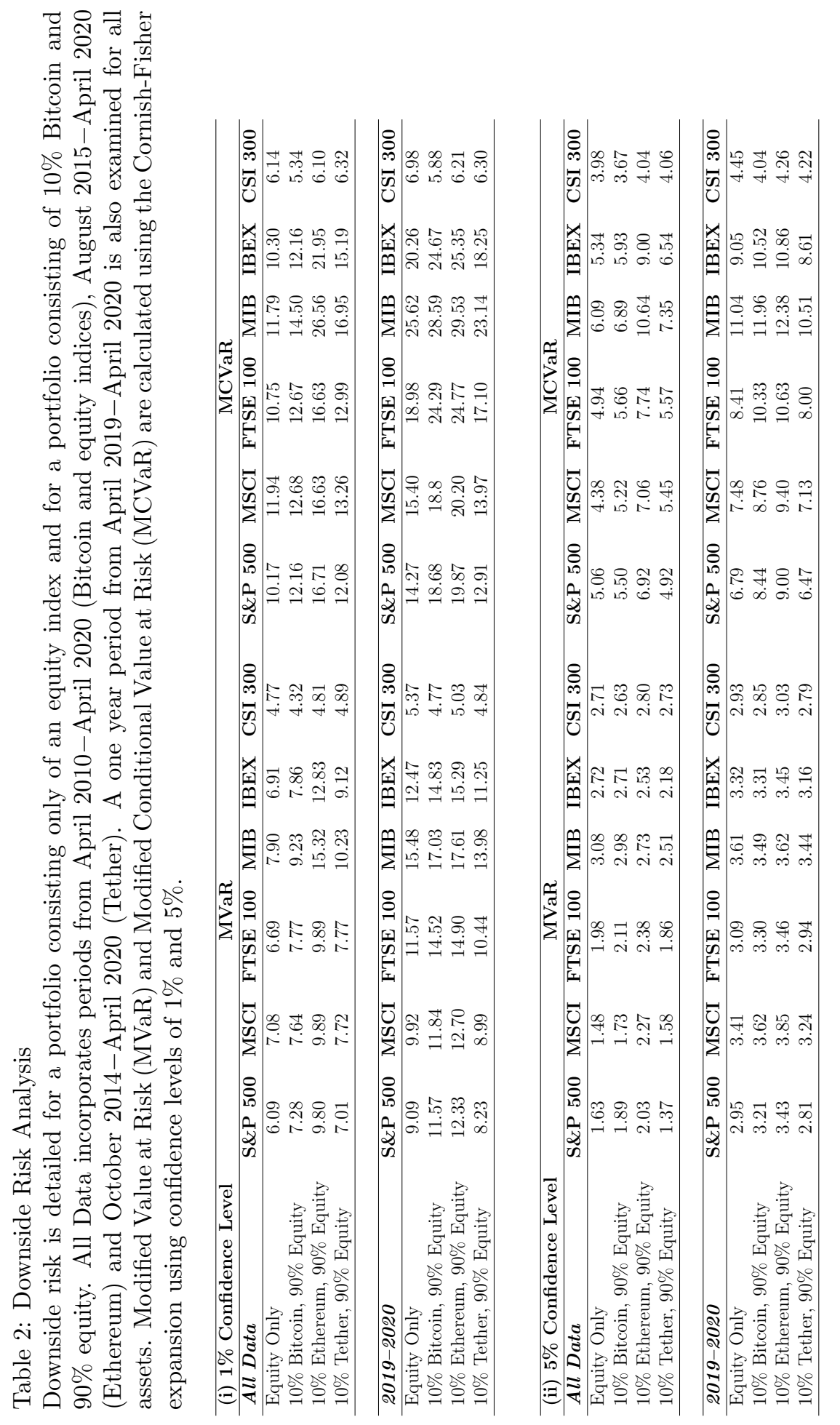




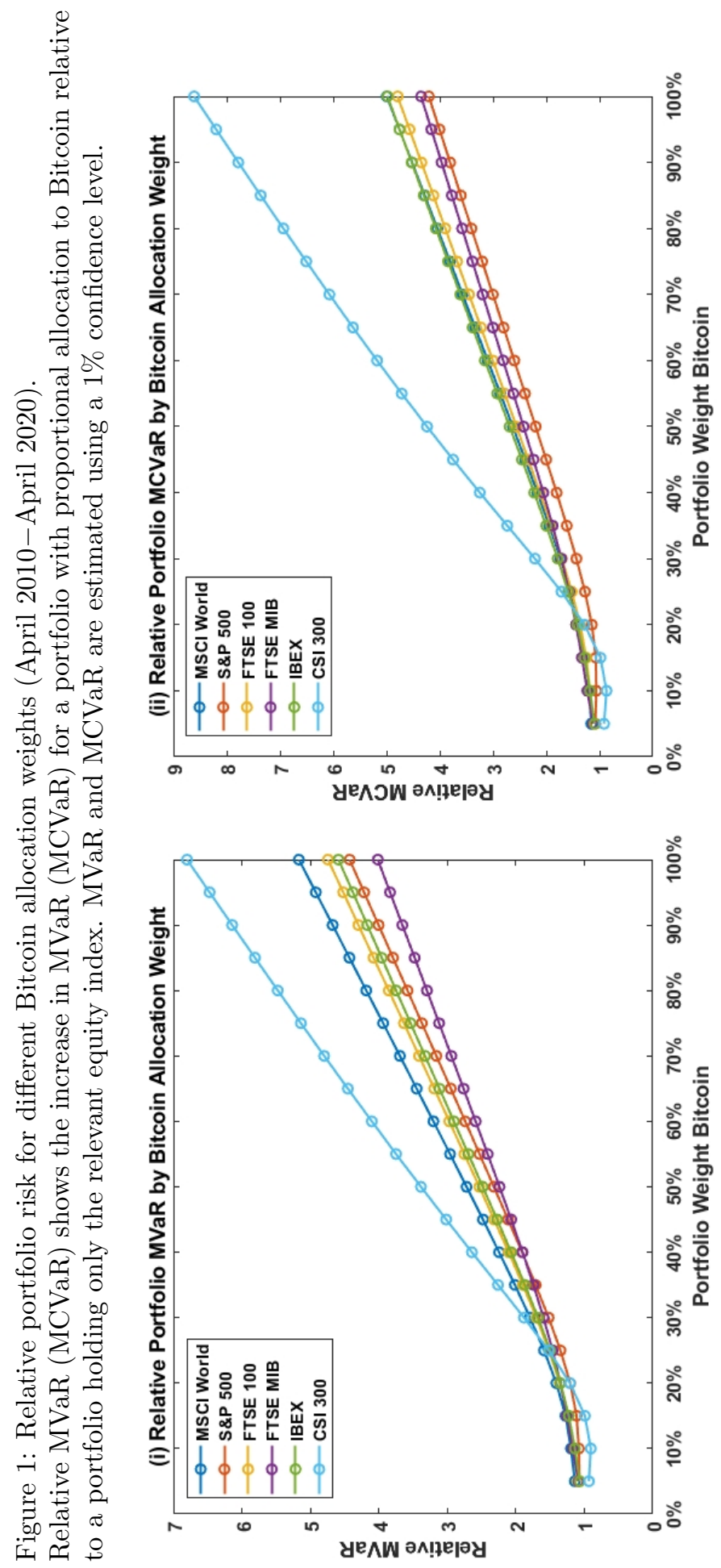




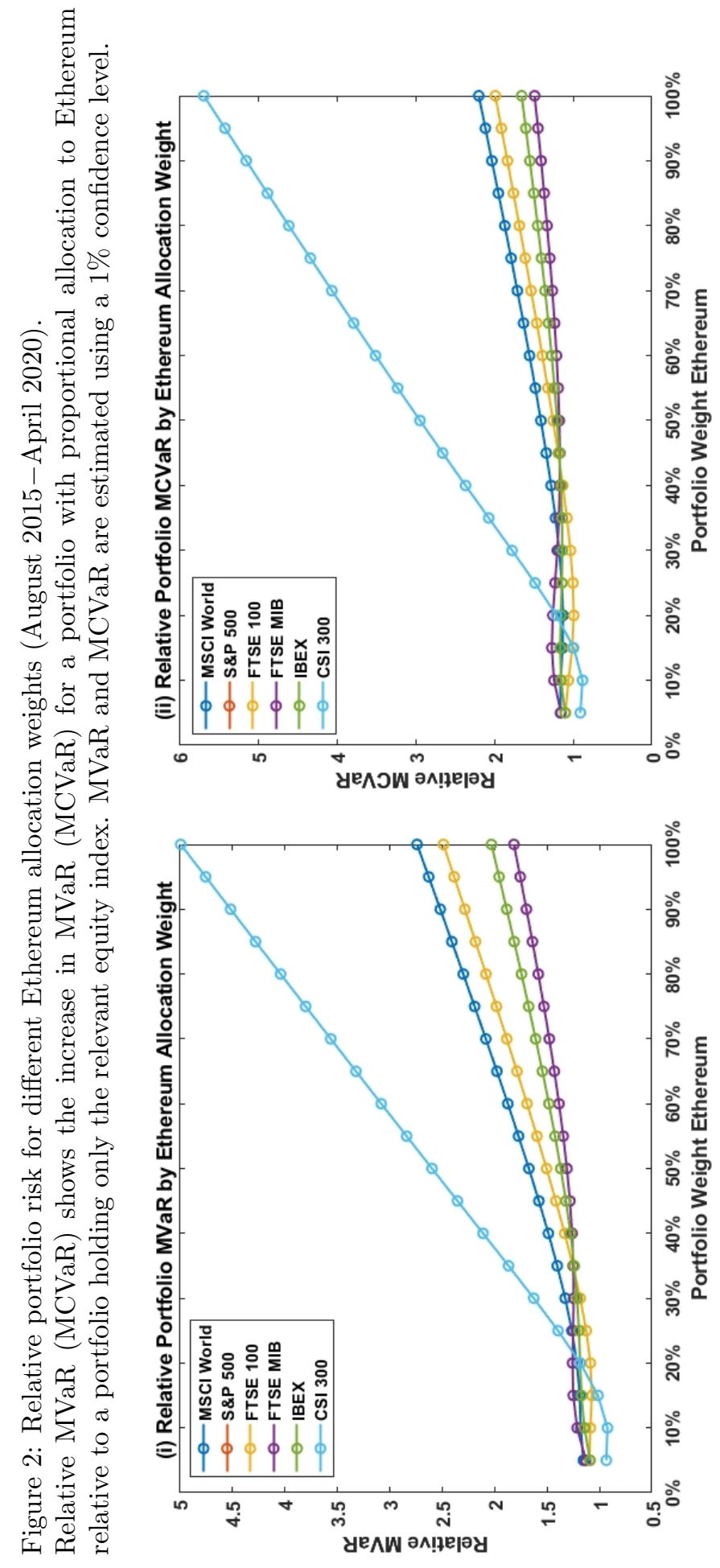




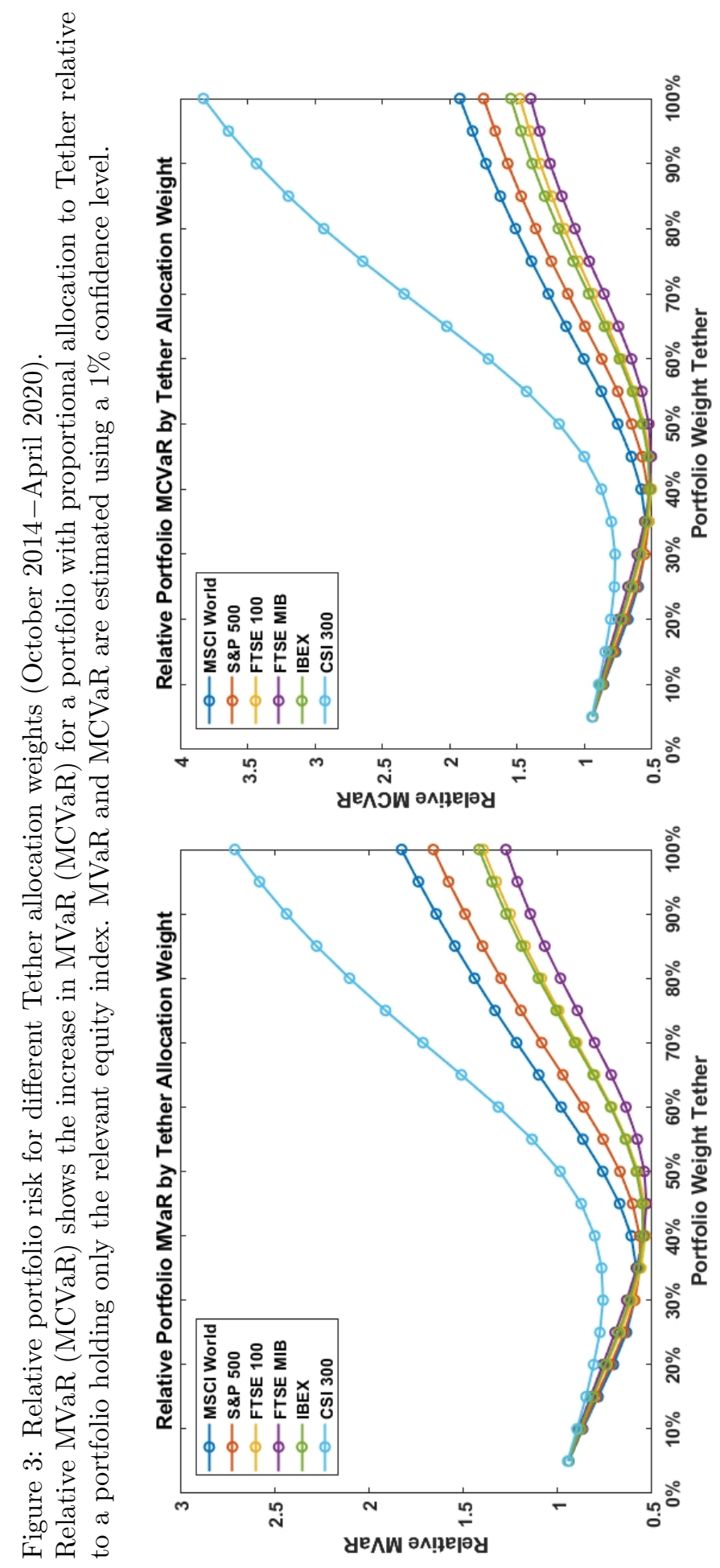


Figure 4: Bitcoin Downside Risk Moving Window Analysis

Relative downside risk is calculated using a one-year moving window for each international market. Relative MVaR (MCVaR) shows the increase in MVaR (MCVaR) for a portfolio holding 10\% Bitcoin and $90 \%$ of the relevant equity index relative to a portfolio holding only the equity index. MVaR and MCVaR are estimated using a $1 \%$ confidence level.
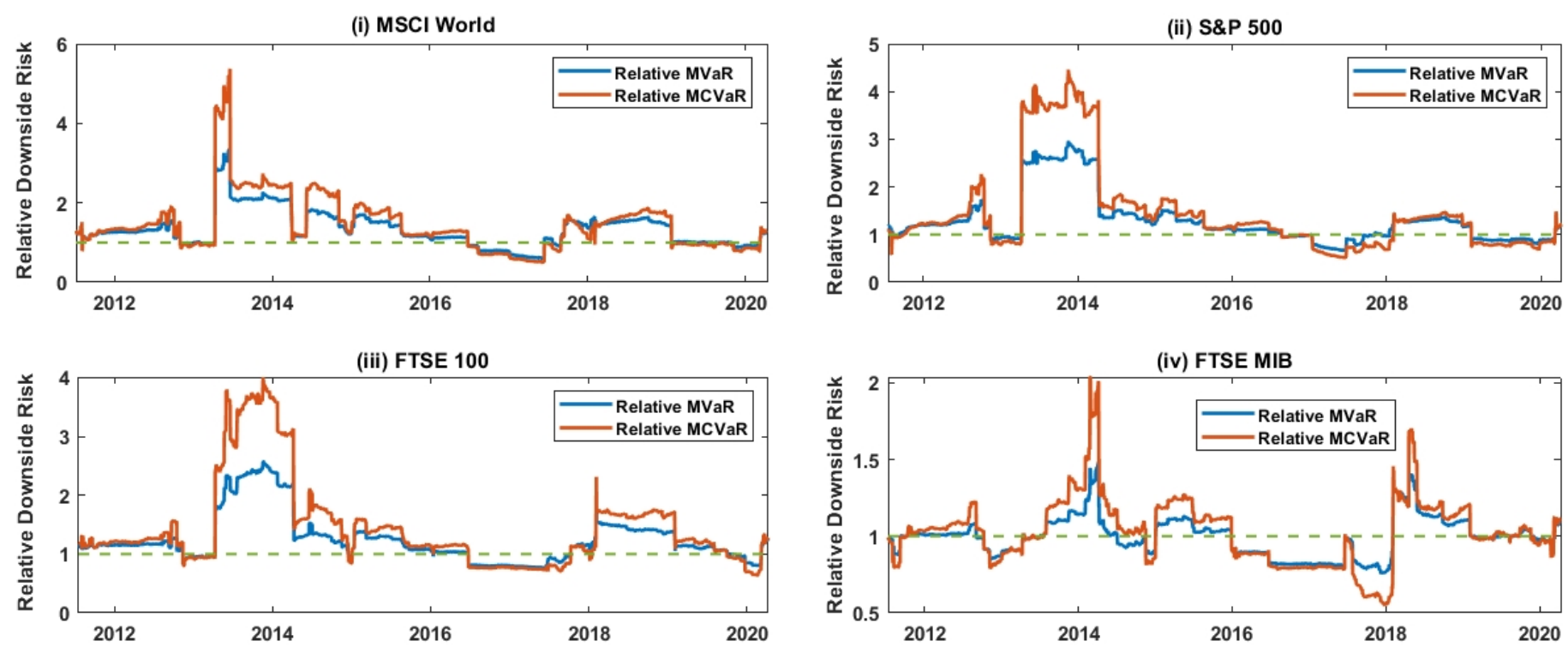

(v) IBEX
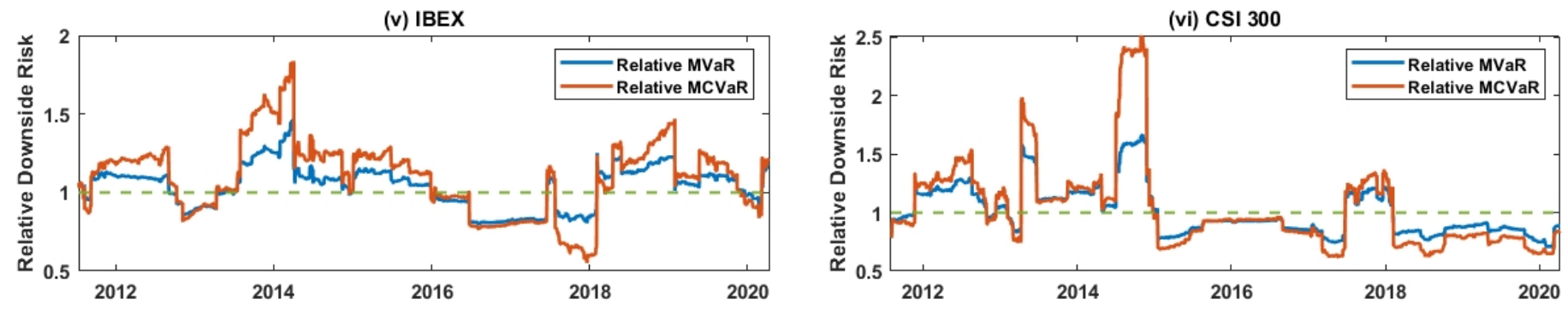
Figure 5: Ethereum Downside Risk Moving Window Analysis

Relative downside risk is calculated using a one-year moving window for each international market. Relative MVaR (MCVaR) shows the increase in MVaR (MCVaR) for a portfolio holding 10\% Ethereum and $90 \%$ of the relevant equity index relative to a portfolio holding only the equity index. MVaR and MCVaR are estimated using a 1\% confidence level.
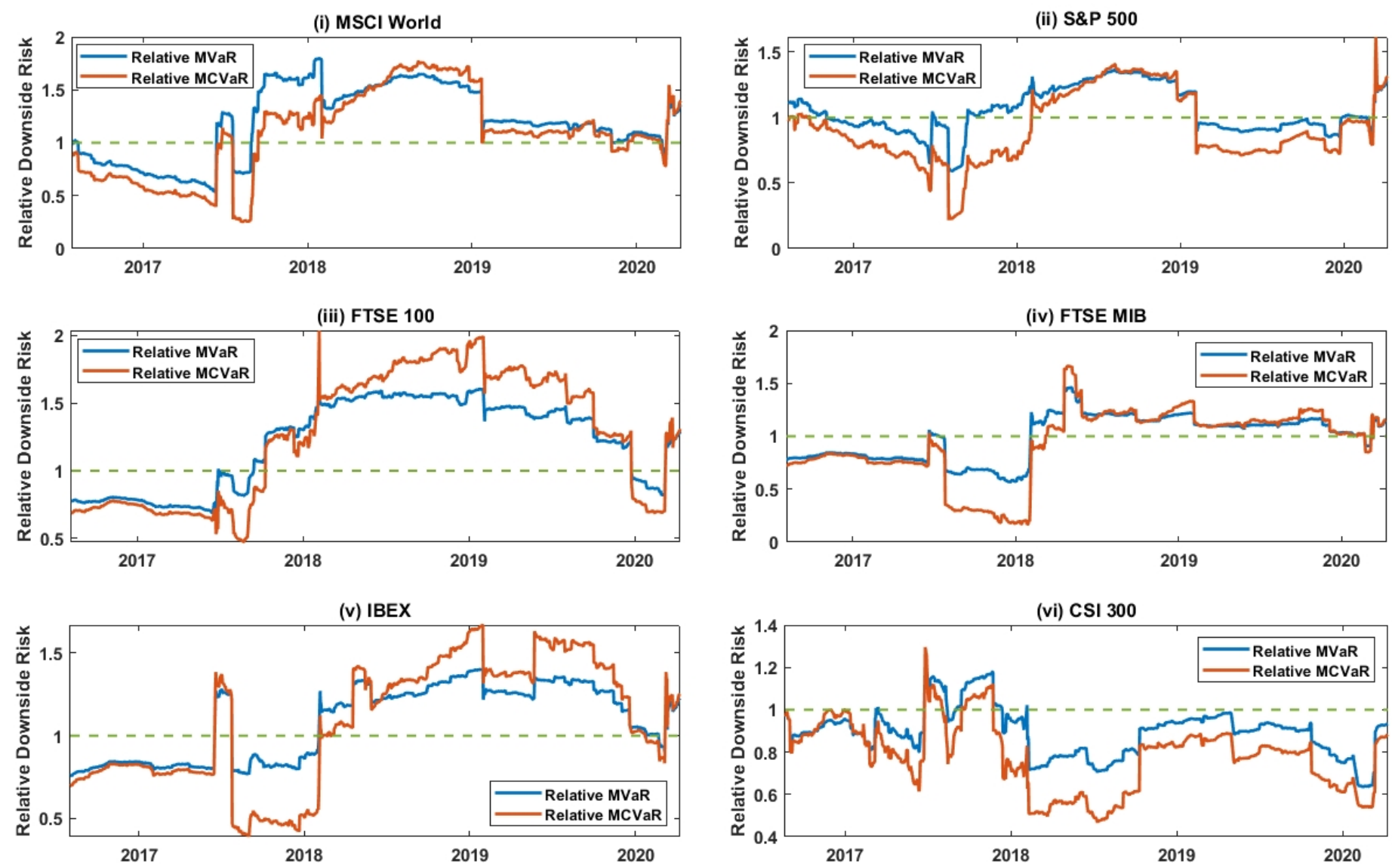
Figure 6: Tether Downside Risk Moving Window Analysis

Relative downside risk is calculated using a one-year moving window for each international market. Relative MVaR (MCVaR) shows the increase in MVaR (MCVaR) for a portfolio holding $10 \%$ Tether and $90 \%$ of the relevant equity index relative to a portfolio holding only the equity index. MVaR and MCVaR are estimated using a $1 \%$ confidence level.
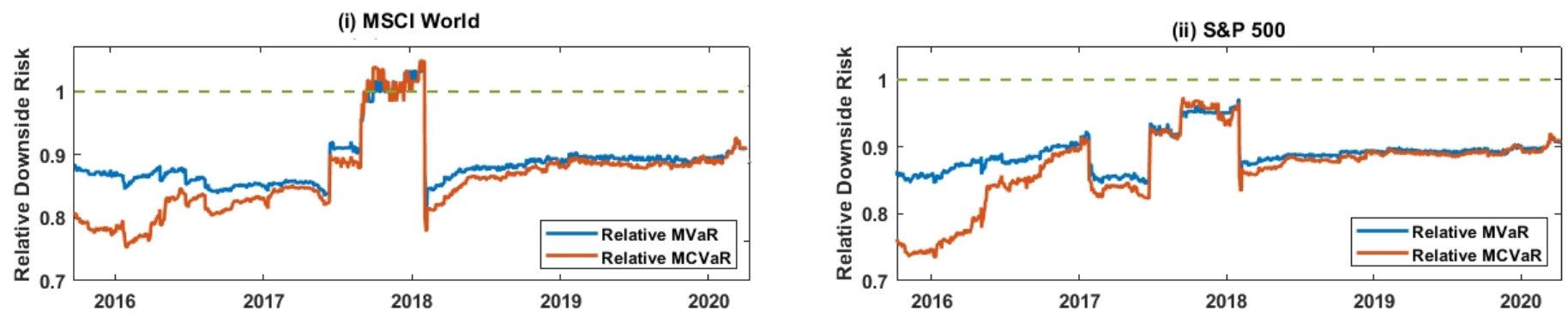

(iii) FTSE 100
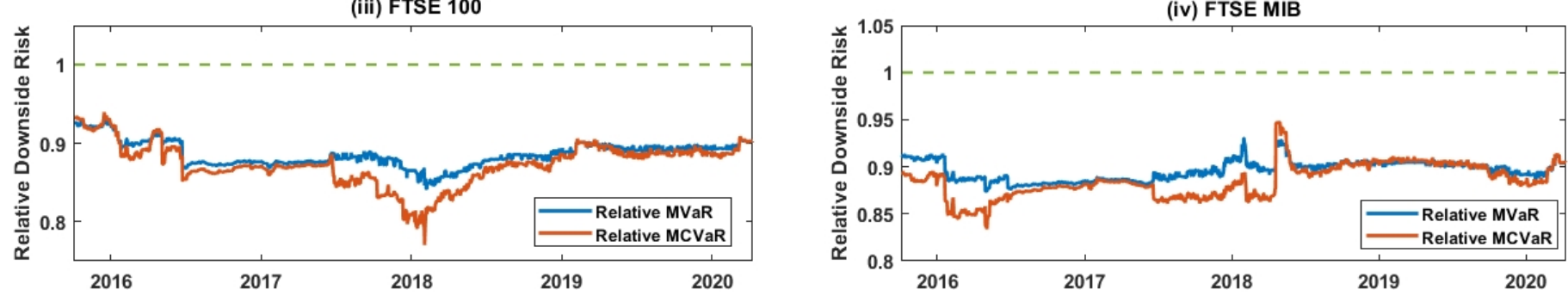

(v) IBEX
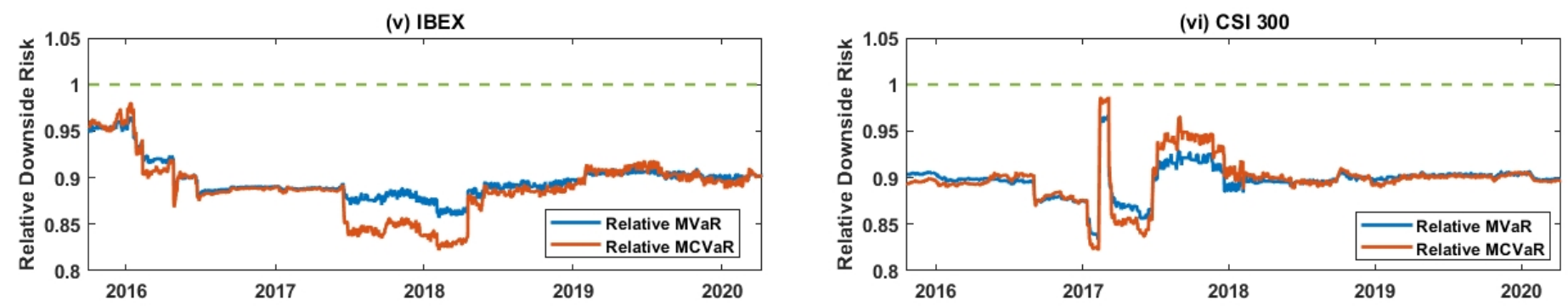